\title{
High peak power sub-60 fs Yb:KGW laser
}

\author{
R. Akbari ${ }^{1}$, K.A. Fedorova ${ }^{2}$, E.U. Rafailov ${ }^{2}$, A. Major ${ }^{1}$ \\ ${ }^{1}$ Department of Electrical and Computer Engineering, University of Manitoba, Winnipeg, R3T 5V6, Canada \\ ${ }^{2}$ Optoelectronics and Biomedical Photonics Group, School of Engineering \& Applied Science, Aston University, UK \\ a.major@umanitoba.ca
}

\begin{abstract}
A high power sub-60 fs mode-locked diode-pumped Yb:KGW laser based on hybrid action of an InGaAs quantum-dot saturable absorber mirror and Kerr-lens mode locking was demonstrated. The laser delivered 56 fs pulses with $1.95 \mathrm{~W}$ of average power corresponding to $450 \mathrm{~kW}$ of peak power. The spectral bandwidth of the pulse was $20.5 \mathrm{~nm}$, which was near the gain bandwidth limit of the Yb:KGW crystal. To the best of our knowledge, these are the shortest pulses generated from the monoclinic double tungstate crystals (and Yb:KGW laser crystal in particular) and the most powerful in the sub-60 fs regime. At the same time they are also the shortest pulses produced to date with the help of a quantum-dot-based saturable absorber.
\end{abstract}

Keywords - femtosecond pulses; passive mode locking; Ybdoped crystal; diode-pumped laser.

\section{INTRODUCTION}

The Yb-ion based laser oscillators [1-8] are well suited for generation of high power ultrashort pulses for a variety of nonlinear optical experiments [9-17]. Our recent works based on dual action of saturable absorber and Kerr lensing effect (KLAS) proposed an effective mechanism for benefiting from selfstarting operation and fast modulation operation of both effects [18-20]. Employing a QD-SESAM offered a promising performance, because it exhibits sub-picosecond recovery time, low saturation fluence and broadband operation [21]. In this work, we demonstrated the generation of 56 fs pulses from a diode-pumped $\mathrm{Yb}: \mathrm{KGW}$ laser oscillator based on KLAS mode locking mechanism.

\section{EXPERIMENTAL SETUP AND RESULTS}

The laser used a $6 \mathrm{~mm}$-long 1.6 at.\% Yb:KGW crystal (cut along the $\mathrm{N}_{\mathrm{g}}$-axis) in a Z-fold cavity. Thermal lensing was taken into account [22,23]. A fiber-coupled laser diode with a maximum power of $30 \mathrm{~W}$ at $980 \mathrm{~nm}$ was used as a pump and the laser delivered up to $6 \mathrm{~W}$ of output power in $\mathrm{CW}$ regime. To initiate the mode locking process, a QD-SESAM (Innolume $\mathrm{GmbH}$ ) was used which was grown with 5 pairs of InGaAs quantum dot layers in the saturable structure and had $0.5 \%$ modulation depth with a saturation fluence of $25 \mu \mathrm{J} / \mathrm{cm}^{2}$. By providing a total GVD of $-3200 \mathrm{fs}^{2}$ using GTI mirrors and adjusting the cavity mode-size for introducing the Kerr lensing effect, $56 \mathrm{fs}$ pulses with an average output power of $1.95 \mathrm{~W}$ at the repetition rate of $77.3 \mathrm{MHz}$ were generated for an incident pump power of $18.3 \mathrm{~W}$, corresponding to $450 \mathrm{~kW}$ of peak power.

A dispersive wave accompanied the mode-locked laser at $1081 \mathrm{~nm}$, which lies in the normal regime when considering the total dispersion in the cavity and phase-matching condition with the main pulse [24]. The presence of the dispersive wave did not affect the mode-locked laser. In order to eliminate the dispersive wave, flatter and broader anomalous GVD profile per round-trip for the intended wavelength range is required.
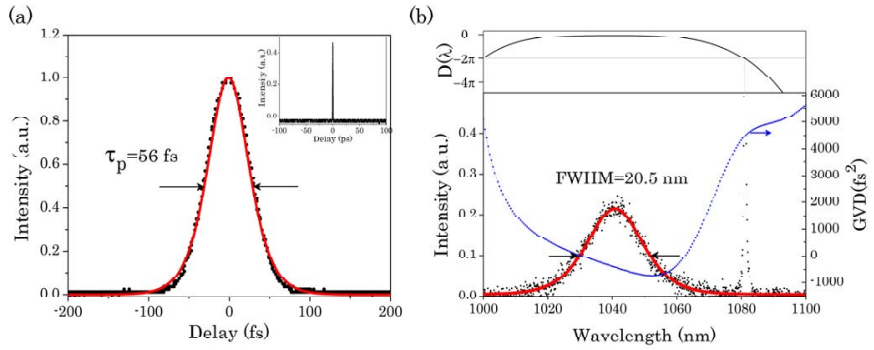

Fig 1. (a) The intensity autocorrelation of the mode-locked laser. Inset: a widerange scan (200 ps). (b) The spectrum of the mode-locked laser (black dotted curve), the calculated round-trip GVD (blue dotted curve) and the phase matching dispersive function $\mathrm{D}(\lambda)$ (upper graph). The $\mathrm{sech}^{2}$-shape fits are shown as red solid curves.

In summary, the generation of high power 56 fs pulses from a diode-pumped $\mathrm{Yb}: \mathrm{KGW}$ was demonstrated. These are the shortest pulses generated to date from the monoclinic double tungstate crystals. The average output and peak powers of the laser are an order of magnitude higher than the previously reported values in the sub-60 fs regime.

\section{REFERENCES}

[1] A. Major, D. Sandkuijl, and V. Barzda, Laser Phys. Lett. 6, 779 (2009).

[2] A. Major, R. Cisek, and V. Barzda, Proc. SPIE 6108, 61080Y (2006)

[3] A. Major, L. Giniunas, N. Langford, A.I. Ferguson, D. Burns, E. Bente, and R. Danielius, J. Mod. Opt. 49, 787 (2002).

[4] R. Akbari, H. Zhao, and A. Major, Opt. Lett. 41, 16014 (2016)

[5] H. Zhao and A. Major, Appl. Phys. B 122, 163 (2016).

[6] H. Zhao and A. Major, Laser Phys. 23, 95001 (2013).

[7] H. Mirzaeian, S. Manjooran, and A. Major, Proc. SPIE 9288, 928802 (2014).

[8] H. Zhao and A. Major, Opt. Express 22, 26651 (2014).

[9] R. Akbari and A. Major, Laser Phys. 23, 35401 (2013).

[10] H. Zhao, I.T. Lima, and A. Major, Laser Phys. 20, 1404 (2010).

[11] S. Manjooran, H. Zhao, I. T. Lima, A. Major, Laser Phys. 22, 1325 (2012).

[12] I.T. Lima Jr., V. Kultavewuti, and A. Major, Laser Phys. 20, 270 (2010).

[13] A. Major, D. Sandkuijl, and V. Barzda, Opt. Express 17, 12039 (2009).

[14] D. Sandkuijl, R. Cisek, A. Major, and V. Barzda, Biomed. Opt. Express 1, $895(2010)$

[15] A. Major, J. S. Aitchison, P. W. E. Smith, N. Langford, and A. I. Ferguson, Opt. Lett. 30, 421 (2005).

[16] A. Major, F. Yoshino, J. S. Aitchison, P. W. E. Smith, E. Sorokin, and I. T. Sorokina, Appl. Phys. Lett. 85, 4606 (2004).

[17] I.P. Nikolakakos, A. Major, J.S. Aitchison, P.W.E. Smith, IEEE J. Sel. Top. Quantum Electron. 10, 1164 (2004).

[18] R. Akbari, H. Zhao, K. A. Fedorova, E. U. Rafailov, and A. Major, Opt. Lett. 41, 3771 (2016).

[19] H. Zhao and A. Major, Opt. Express 22, 30425 (2014).

[20] R. Akbari, K. A. Fedorova, E. U. Rafailov, and A. Major, Appl. Phys. B 123, 123 (2017).

[21] A. A. Lagatsky, et al., Prog. Quantum Electron. 34, 1 (2010).

[22] P. Loiko, S. Manjooran, K. Yumashev, and A. Major, Appl. Opt. 56, 2937 (2017).

[23] T. Waritanant and A. Major, Appl. Phys. B 122, 135 (2016).

[24] Q. Lin, et al., Opt. Commun. 153, 285 (1998). 